\title{
Exceptionality and Natural Language Learning
}

\author{
Mihai Rotaru \\ Diane J. Litman \\ Computer Science Department \\ University of Pittsburgh \\ Pittsburgh, PA 15260 \\ mrotaru, litman@cs.pitt.edu
}

\begin{abstract}
Previous work has argued that memory-based learning is better than abstraction-based learning for a set of language learning tasks. In this paper, we first attempt to generalize these results to a new set of language learning tasks from the area of spoken dialog systems and to a different abstraction-based learner. We then examine the utility of various exceptionality measures for predicting where one learner is better than the other. Our results show that generalization of previous results to our tasks is not so obvious and some of the exceptionality measures may be used to characterize the performance of our learners.
\end{abstract}

\section{Introduction}

Our paper is a follow-up of the study done by Daelemans et al. (1999) in which the authors show that keeping exceptional training instances is useful for increasing generalization accuracy when natural language learning tasks are involved. The tasks used in their experiments are: grapheme-phoneme conversion, part of speech tagging, prepositional phrase attachment and base noun phrase chunking. Their study provides empirical evidence that editing exceptional instances leads to a decrease in memory-based learner performance. Next, the memory-based learner is compared on the same tasks with a decision-tree learner and their results favor the memory-based learner. Moreover, the authors provide evidence that the performance of their memory-based learner is linked to its property of holding all instances (including exceptional ones) and general properties of language learning tasks (difficultness in discriminating between noise and valid exceptions and sub-regularities for those tasks).

We continue on the same track by investigating if their results hold on a different set of tasks. Our tasks come from the area of spoken dialog systems and have smaller datasets and more features (with many of the features being numeric, in contrast with the previous study that had none). We observe in our experiments with these tasks a much smaller exceptionality measure range compared with the previous study. Our results indicate that the previous results do not generalize to all our tasks.

An additional goal of our research is to investigate a new topic by looking into whether exceptionality measures can be used to characterize the performance of our learners: a memory-based learner (IB1-IG) and a rulebased learner (Ripper). Our results indicate that for some of the exceptionality measures we will examine, IB1-IG is better for predicting typical instances while Ripper is better for predicting exceptional instances.

We will use the following conventions throughout the paper. The term "exceptional" will be used to label instances that do not follow the rules that characterize the class they are part of (in language learning terms, they are "bad" examples of their class rules). We will use "typical" as the antonym of this term; it will label instances that are good examples of their class rules. The fact that an instance is typical should not be confused with an exceptionality measure we will use that has the same name (typicality measure).

\section{Learning methods}

We will use in our study the same memory-based learner that was used in the previous study: IB1-IG. The abstraction-based learner used in the previous study was C5.0 (a commercial implementation of the $\mathrm{C} 4.5$ decision tree learner). In our study we will use a rule-based learner, Ripper. Although the two abstraction-based learners are different, they share many features (many techniques used in rule-based learning have been adapted from decision tree learning (Cohen, 1995)) ${ }^{1}$.

\footnotetext{
${ }^{1}$ We used Ripper because its implementation was available and previous studies on our language learning tasks were performed using Ripper
} 


\subsection{IB1-IG}

Our memory-based learner is called IB1-IG and is part of TiMBL, a software package developed by the ILK Research Group, Tilburg University and the CNTS Research Group, University of Antwerp. TiMBL is a collection of memory-based learners that sit on top of the classic k-NN classification kernel with added metrics, algorithms, and extra functions.

Memory-based reasoning is based on the hypothesis that humans, in order to react to a new situation, first compare the new situation with previously encountered situations (which reside in their memory), pick one or more similar situations, and react to the new one based on how they reacted to those similar situations. This type of learning is also called lazy learning because the learner does not build a model from the training data. Instead, typically, the whole training set is stored. To predict the class for a new instance, the lazy learner compares it with stored instances using a similarity metric and the new instance class is determined based on the classes of the most similar training instances. At the algorithm level, lazy learning algorithms are versions of $\mathrm{k}$-nearest neighbor (k-NN) classifiers.

IB1-IG is a k-NN classifier that uses a weighted overlap metric, where a feature weight is automatically computed as the Information Gain (IG) of that feature. The weighted overlap metric for two instances $X$ and $Y$ is defined as:

$$
\Delta(X, Y)=\sum_{i=1}^{n} w_{i} \delta\left(x_{i}, y_{i}\right)
$$

where:

$$
\delta\left(x_{i}, y_{i}\right)=\left\{\begin{array}{cl}
a b s\left(\frac{x_{i}-y_{i}}{\max _{i}-\min _{i}}\right) & \text { if numeric, else } \\
0 & \text { if } x_{i}=y_{i} \\
1 & \text { if } x_{i} \neq y_{i}
\end{array}\right.
$$

Information gain is computed for every feature in isolation by computing the difference in uncertainty between situations with or without knowledge of the feature value (for more information, see Daelemans et al., 2001). These values describe the importance of that feature in predicting the class of an instance and are used as feature weights.

\subsection{Ripper}

Ripper is a fast and effective rule-based learner developed by William Cohen (Cohen, 1995). The algorithm has an overfit-and-simplify learning strategy: first an initial rule set is devised by overfitting a part of the training set (called the growing set) and then this rule set is repeatedly simplified by applying pruning operators and testing the error reduction on another part of the training set (called the pruning set). Ripper produces a model consisting of an ordered set of if-then rules.

There are several advantages to using rule-based learners. The most important one is the fact that people can understand relatively easy the model learned by a rule-based learner compared with the one learned by a decision-tree learner, neural network or memory-based learner. Also, domain knowledge can be incorporated in a rule-based learner by altering the type of rules it can learn. Finally, rule-based learners are relatively good at filtering the potential noise from the training set. But in the context of natural language learning tasks where distinguishing between noise and exceptions and subregularities is very hard, this filtering may result in a decrease in accuracy. In contrast, memory-based learners, by keeping all instances around (including exceptional ones), may have higher classification accuracy for such tasks.

\section{Exceptionality measures}

One of the main disadvantages of memory-based learning is the fact that the entire training set is kept. This leads to serious time and memory performance drawbacks if the training set is big enough. Moreover, to improve accuracy, one may want to have noisy instances present in the training set pruned. To address these problems there has been a lot of work on trying to edit part of the training set without hampering the accuracy of the predictor. Two types of editing can be done. One can edit redundant regular instances (because the training set contains a lot of similar instances for that class) and/or unproductive instances (the ones that present irregularities with respect to the training set space).

There are many measures that capture both types of instances. We will use the ones from the previous study (typicality and class prediction strength) and a new one called local typicality. Even though these measures were devised with the purpose of editing part of the training set, they are used in our study and the previous study to point out instances that should not be removed, at least for language learning tasks.

\subsection{Typicality}

We will use the typicality definition from Daelemans et al. (1999) which is similar to the definition from Zhang (1992). In both cases, a typicality function is defined whose extremes correspond to exceptional and typical instances. The function requires a similarity measure which is defined in both cases as the inverse of the distance between two instances. The difference between the two implementations of typicality is that Zhang (1992) defines the distance as the Euclidian distance while Daelemans et al. (1999) use the normalized 
weighted Manhattan distance from (1). Thus, our similarity measure will be defined as:

$$
\operatorname{sim}(X, Y)=\sum_{i=1}^{n} w_{i}\left(1-\delta\left(x_{i}, y_{i}\right)\right)
$$

For every instance $X$, a subset of the dataset called family of $X, \operatorname{Fam}(X)$, is defined as being all instances from the dataset that have the same class as $X$. All remaining instances form the unrelated instances subset, $\operatorname{Unr}(X)$. Then, intra-concept similarity is defined as the average similarity between $X$ and instances from $\operatorname{Fam}(X)$ and inter-concept similarity as the average similarity between $X$ and instances from $\operatorname{Unr}(X)$.

$$
\begin{aligned}
& \operatorname{Intra}(X)=\frac{1}{|\operatorname{Fam}(X)|} \sum_{i=1}^{|\operatorname{Fam}(X)|} \operatorname{sim}\left(X, \operatorname{Fam}(X)_{i}\right) \\
& \operatorname{Inter}(X)=\frac{1}{|\operatorname{Unr}(X)|} \sum_{i=1}^{|\operatorname{Unr}(X)|} \operatorname{sim}\left(X, \operatorname{Unr}(X)_{i}\right)
\end{aligned}
$$

Finally, typicality of an instance $X$ is defined as the ratio of its intra-concept and inter-concept similarity.

$$
\operatorname{Typicality}(X)=\frac{\operatorname{Intra}(X)}{\operatorname{Inter}(X)}
$$

The typicality values are interpreted as follows: if the value is higher than 1 , then that instance has an intra-concept similarity higher than inter-concept similarity, thus one can say that the instance is a good example of its class (it is a typical instance). A value less than 1 implies the opposite: the instance is not a good example of its class (it is an exceptional instance). Values around 1 are called by Zhang boundary instances since they seem to reside at the border between concepts.

\subsection{Class prediction strength}

Another measure used in the previous study is the class prediction strength (CPS). This measure tries to capture the ability of an instance to predict correctly the class of a new instance. We will employ the same CPS definition used in the previous study (the one proposed by Salzberg (1990)). In the context of k-NN, predicting the class means, typically, that the instance is the closest neighbor for a new instance. Thus the CPS function is defined as the ratio of the number of times our instance is the closest neighbor for an instance of the same class and the number of times our instance is the closest neighbor for another instance regardless of its class. A CPS value of 1 means that if our instance is to influence another instance class (by being its closest neighbor) its influence is good (in the sense that predicting the class using our instance class will result in an accurate prediction). Thus our instance is a good predictor for our class, i.e. it is a typical instance. In contrast, a value of 0 indicates a bad predictor for the class and thus labels an exception instance. A value of 0.5 will correspond to instances at the border between concepts.
Unlike typicality, when computing CPS, we can encounter situations when its value is undefined (zero divided by zero). This means that the instance is not the closest neighbor for any other instance. Since there is no clear interpretation of instance properties in this case, we will set its CPS value to a constant higher than 1 (no particular meaning of the value, just to recognize it in our graphs).

\subsection{Local typicality}

While CPS captures information very close to an instance, typicality as defined by Zhang captures information from the entire dataset. But this may not be the most desirable measure in cases such as those when a concept is made of at least two disjunctive clusters. Consider the example from Figure 1. For an instance in the center of cluster $A_{1}$, its similarity with instances from the same cluster is very high but very low with instances from cluster $\mathrm{A}_{2}$. At the same time, its similarity with instances from class $\mathrm{B}$ is somewhere between above two values. When everything is averaged, instance intra-concept and inter-concept similarity have comparable values thus leading to a typicality value around 1 even if the instance is highly typical for the cluster $\mathrm{A}_{1}$.

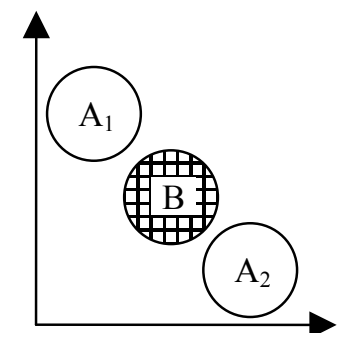

Figure 1. Class distribution that causes flattening in typicality distribution

To address this problem, we changed the definition of $\operatorname{Fam}(X)$ and $\operatorname{Unr}(X)$. Instead of considering all instances from the dataset when building the two subsets, we will be using only instances from a vicinity of our instance. The typicality computed using these new subsets will be called local typicality. To define the vicinity, we used again the similarity metric. When two instances are identical, their similarity has the maximum value which is the sum of all feature weights. An instance is in the vicinity of another instance if and only if their similarity has a value higher than a given percent of maximum similarity value (using this definition of vicinity instead of a specified number of nearest neighbors, makes our exceptionality measure adaptive to the density of the local neighborhood). For our datasets, a percent value of $90 \%$ yields the best results furnishing a measure that is different from both typicality and CPS.

Like CPS, division by zero can appear when computing local typicality. This means that inter-concept 
similarity is zero and this can only happen if there is no instance with a different class in the vicinity of our instance. In this case, if the intra-concept similarity is higher than 0 (there is at least one instance from the same class in the vicinity) we set the local typicality to a maximum value, while if the intra-concept similarity is 0 , then we set the typicality to a minimum value (no one in the vicinity of this instance is a good indication of an exceptional instance). When inter-concept similarity is higher than 0 , we will set the local typicality to a minimum value if its intra-concept similarity is 0 (so that we will not have a big gap between local typicality values). Minimum and maximum values are computed as values to the left and right of the local typicality interval for non-exceptional cases.

We can rank our exceptionality measures by the level of information they capture (from most general to most local): typicality, local typicality and CPS.

\section{Language learning tasks}

The tasks we will be using in our study come from the area of spoken dialog systems (SDS). They were all designed as methods for potentially improving the dialog manager of a SDS system called TOOT (Litman and Pan, 2002). This system provides access to train information from the web via telephone and it was developed for the purpose of comparing differences in dialog strategy.

Our tasks are: (1) Identifying user corrections (ISCORR), (2) Identifying correction-aware sites (STATUS), (3) Identifying concept-level speech recognition errors (CABIN) and (4) Identifying word-level speech recognition errors (WERBIN). The first task is a binary classification task that labels each user turn as to whether or not it is an attempt from the user to correct a prior system recognition failure. The second task is a 4way classification task that extends the previous one with whether or not the user is aware the system made a recognition error. The four classes are: normal user turn, user only tries to correct the system, user is only aware of a system recognition error, and user is both aware of and tries to correct the system error. The third and the fourth tasks are binary classification tasks that try to predict the system speech recognition accuracy when recognizing a user turn. CABIN measures a binary version of the Concept Accuracy (percent of semantic concepts recognized correctly) while WERBIN measures a binary version of the Word Error Rate (percent of words recognized incorrectly).

Data for our tasks was gathered from a corpus of 2,328 user turns from 152 dialogues between human subjects and TOOT. The features used to represent each user turn include prosodic information, information from the automatic speech recognizer, system conditions and dialog history. Then, each user turn was la- beled with respect to every classification task. Even though our classification tasks share the same data, there are clear differences between them. ISCORR and STATUS both deal with user corrections which is quite different from predicting speech recognition errors (handled in WERBIN and CABIN). Moreover, one will expect very little noise or no noise at all when manually annotating WERBIN and CABIN. For more information on our tasks and features, see (Litman et al., 2000; Hirschberg et al., 2001; Litman et al., 2001).

There are a number of dimensions where our tasks differ from the tasks from the previous study. First of all our datasets are smaller (2,328 instances compared with at least 23,898). Second, the number of features used is much bigger than the previous study (141 compared with 4-11). Moreover, many features from our datasets are numeric while the previous study had none. These differences will also reflect on our exceptionality measures values. For example, the smallest range for typicality in the previous study was between 0.43 and 10.57 while for our tasks it is between 0.9 and 1.1. To explore these differences we varied the feature set used. Instead of using all the available features (this feature set is called $A l l$ ), we restricted the feature set by using only non-numeric features (Nonnum - 22 features). The typicality range increased when using this feature set $(0.77$ 1.45 ), but the number of features used was still larger than the previous study. For this reason, we next devised two set of features with only 9 (First 9 ) and 15 features (First15). The features were selected based on their information gain (see section 2.1).

Before proceeding with our results, there is one more thing we want to mention. At least half of our instances have one or more missing values and while the Ripper implementation offered a way to handle them, there was no default handling of missing values in the IB1-IG implementation. Thus, we decided to replace missing values ourselves before presenting the datasets to our learners. In particular there are two types of missing values: genuine missing values (no value was provided; we will refer to them as missing values) and undefined values. Undefined values come from features that are not defined in that user turn (for example, in the first user turn, most of the dialog history features were undefined because there was no previous user turn).

For symbolic features, we replaced missing and undefined values with a given string for missing values and another one for undefined values. For numeric features, the problem was more complicated since the distance metric uses the difference between two numeric values and thus, the values used to fix the problem can influence the distance between instances. We experimented with different replacement values: to the left and right of the interval boundaries for that features, both replacement values on one side of the interval or very far from the interval boundaries. All experiments with 
the values provided comparable results. For our experiments, missing values were replaced with a value to the right of the interval for that feature and undefined values were replaced with a value to the left of that interval.

\section{Results}

In 5.1 we reproduce the editing and comparison experiments from the previous study to see if their results generalize to our tasks. In 5.2, we move to our next goal: characterizing learners' performance using exceptionality measures. Both learners were run using default parameters ${ }^{2}$.

\subsection{Natural language learning and memory- based learning}

First, we performed the editing experiments from the previous study. The purpose of those experiments was to see the impact of editing exceptional and typical instances on the accuracy of the memory-based learner. Since our datasets were small, unlike the previous study which performed editing only on the first train-test partition of a 10-fold cross validation, we performed the editing experiment on all partitions of a 10-fold cross validation. For every fold, we edited $0,1,2,5,10,20$, 30,40 and $50 \%$ of the training set based on extreme values of all our exceptionality criteria. Accuracy after editing a given percent was averaged among all folds (there is a significant difference in accuracies among folds but all folds exhibit a similar trend with the average). Figure 2 shows our results for the ISCORR dataset

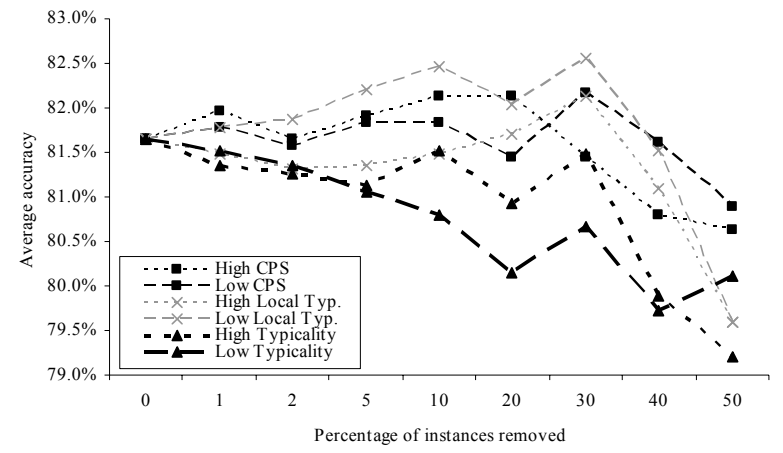

Figure 2. IB1-IG average accuracy after editing a given percent of the training set based on high and low extremes of all exceptionality measures (ISCORR dataset with all features)

\footnotetext{
${ }^{2}$ We performed parameter tuning experiments for both predictors: for every fold of a 10 -fold cross validation, part of the training set was used as a validation set (for tuning parameters). Our results indicate that the tuned parameters depend on the fold used and there was no clear gain to accuracy from tuning (in some cases there was even loss in accuracy). Integrating tuned parameters with our leave-one-out experiments presents additional problems.
}

using six types of editing (editing based on low and high value for all three criteria). In contrast with the previous study, where for all tasks even the smallest editing led to significant accuracy decreases, for our task there was no clear decrease in performance. Moreover, for some criteria (like low local-typicality) we can even see an initial increase in performance. Only after editing half of the training set is there a clear decrease in performance for all editing criteria on this task.

Editing experiments for the other dataset-feature set combinations yield similar results.

Next, we compared the memory-based learner with our abstraction-based learner on all tasks. Since the datasets were relatively small, we performed leave-oneout cross validations. Table 1 summarizes our results. The baseline used is the majority class baseline. First, we run the predictors on all tasks using all features. In contrast with the previous study which favored the memory-based learner for almost all their tasks, our results favor IB1-IG for only two of the four tasks (ISCORR and STATUS). In Section 4, we mentioned that the typicality range for our tasks was very small compared with the previous study. Contrary to what we

\begin{tabular}{|l|c|c|c|c|}
\cline { 2 - 4 } \multicolumn{1}{c|}{} & \multicolumn{3}{c|}{ Error rate } & \multirow{2}{*}{$\begin{array}{c}\text { Typicality } \\
\text { range }\end{array}$} \\
\hline Data-Feat. set & IB1-IG & Ripper & Baseline & $0.94-1.06$ \\
\hline Iscorr-All & $\mathbf{1 4 . 9 9 \%}$ & $16.15 \%$ & $28.99 \%$ & $0.94 \%$ \\
\hline Status-All & $\mathbf{2 2 . 2 5 \%}$ & $23.71 \%$ & $43.04 \%$ & $0.96-1.10$ \\
\hline Cabin-All & $13.10 \%$ & $\mathbf{1 2 . 1 1 \%}$ & $30.50 \%$ & $0.90-1.12$ \\
\hline Werbin-All & $17.65 \%$ & $\mathbf{1 1 . 9 0 \%}$ & $39.22 \%$ & $0.90-1.10$ \\
\hline Iscorr-Nonnum & $17.01 \%$ & $\mathbf{1 6 . 2 4 \%}$ & $28.99 \%$ & $0.81-1.49$ \\
\hline Status-Nonnum & $23.93 \%$ & $\mathbf{2 1 . 9 9 \%}$ & $43.04 \%$ & $0.88-1.62$ \\
\hline Iscorr-First9 & $17.78 \%$ & $\mathbf{1 6 . 0 7 \%}$ & $28.99 \%$ & $0.86-1.17$ \\
\hline Iscorr-First15 & $\mathbf{1 4 . 6 9 \%}$ & $14.95 \%$ & $28.99 \%$ & $0.88-1.14$ \\
\hline
\end{tabular}

Table 1. IB1-IG, Ripper and majority class baseline error rate on some of our dataset-feature set combinations

expected, the tasks where IB1-IG performed better were the ones with smaller typicality range. To investigate the typicality range impact on our predictors, we tried to make our datasets similar to the datasets from the previous study by tackling the feature set. We eliminated all numeric features (since the tasks from the previous study had none) and performed experiments on the tasks that had the less typicality range (again, ISCORR and STATUS). Again, when typicality range was increased, even though there were no numeric features, IB1-IG performed worse than Ripper. IB1-IG error rate increased when using only non-numeric features for both tasks compared with the error rate when using all features. This observation led us to assume that, at least for IB1-IG, some of the relevant features for classification were numeric and they were not present in our feature set. Thus, we selected two sets of features (First9 and First15) based on the features' relevance and performed the experiments again on the ISCORR dataset. We can 
observe that as the number of relevant features is increased, the error rate for both predictors and the typicality range are decreasing and IB1-IG takes the lead when the First 15 feature set is used. Our results indicate that the predictor that performs better depends on the task, the number of features and the type of features we use.

To explore why the previous study's results do not generalize in our case, we are planning to replicate these experiments on the dialog-act tagging task on the Switchboard corpus (a task more similar in size and feature types with the previous study than our tasks but still in the area of spoken dialog systems - see Shriberg et al. (1998)).

\subsection{Characterizing learners' performance using exceptionality measures}

The next goal of our study was to see if we can characterize the performance of our predictors on various classes of instances defined by our exceptionality criteria. In other words, we wanted to try to answer questions like: is IB1-IG better at predicting exceptional instances than Ripper? How about typical instances? Can we combine the two learners and select between them based on the instance exceptionality?

To answer these questions, we performed the leaveone-out experiments described above and recorded for every instance whether our predictors predicted it correctly or incorrectly. Next, we computed the exceptionality of every instance using all three measures. Figure 3 shows the exceptionality distribution using the typicality measure for the ISCORR dataset with all features ${ }^{3}$. The

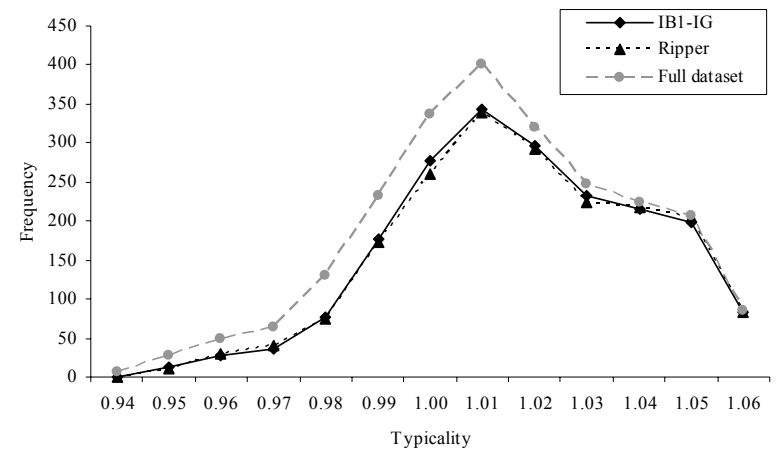

Figure 3. Typicality distribution for all instances, instances correctly predicted by IB1-IG and instances correctly predicted by Ripper (ISCORR dataset with all features)

typicality distributions of all instances from the ISCORR dataset, of instances correctly predicted by IB1-IG, and of instances correctly predicted by Ripper are plotted in the figure. The graph shows that for this dataset there are a lot of boundary instances, very few exceptional instances and few typical instances. The

\footnotetext{
${ }^{3}$ For other dataset-feature set combination graphs see: http://www.cs.pitt.edu/ mrotaru/exceptionality
}

typicality range for all our datasets (usually between 0.85 and 1.15$)$ is far less than the one from the previous study ( 0.43 up to 10 or even 3500 ). According to Zhang (1992) hard concepts are often characterized by small typicality spread. Moreover, small typicality spread is associated with low accuracy in predicting.

Figure 4 shows the same information as Figure 3, but instead of plotting the count, we plot the percentage of the instances with typicality between a given interval that have been correctly classified by one of the predictors. We can observe that accuracy of both predictors increases with typicality. That is, the more typical the

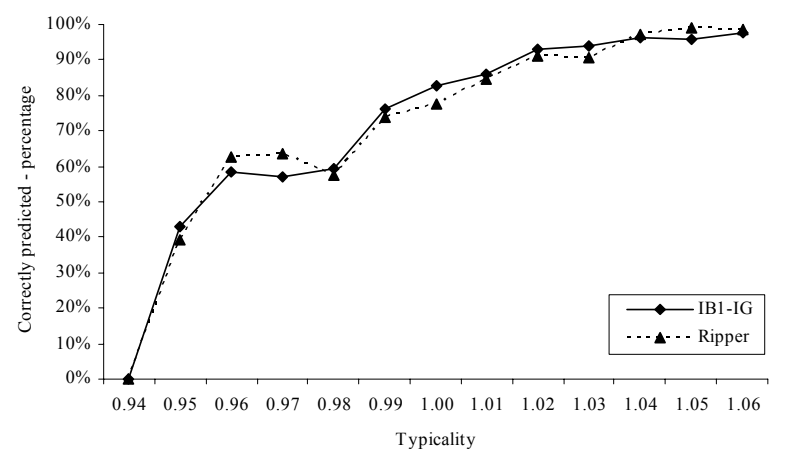

Figure 4. Percent of instances predicted correctly by IB1-IG and Ripper based on instance typicality (ISCORR dataset with all features)

instance, the more reliable the prediction; the more exceptional the instance, the more unreliable the prediction. This observation holds for all our dataset-feature set combinations. It is not clear for the ISCORR dataset whether one predictor is better than the other based on the typicality. But for datasets CABIN and WERBIN where, overall, IB1-IG did worse than Ripper, the same graph (see Figure 5) shows that IB1-IG's accuracy is worse than Ripper's accuracy when predicting low typicality instances ${ }^{4}$. Given the problems with typicality if the concepts we want to learn are clustered, we decided

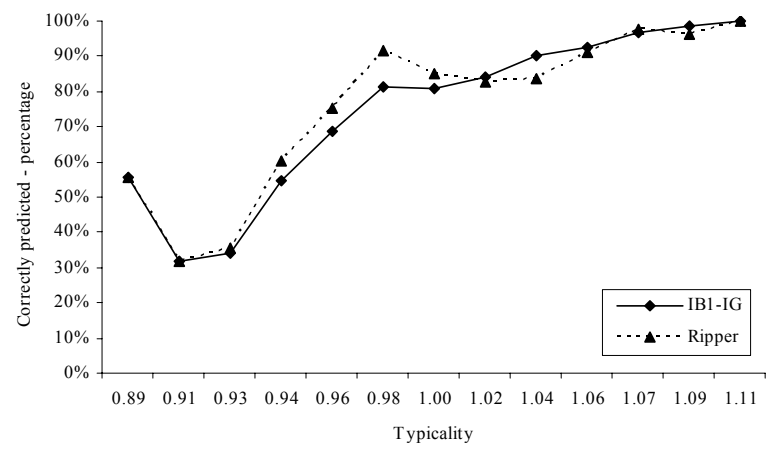

Figure 5. Percent of instances predicted correctly by IB1-IG and Ripper based on instance typicality (CABIN dataset with all features)

\footnotetext{
${ }^{4}$ It was not our point to investigate statistical significance of this trend. As we will see later, this trend is powerful enough to yield interesting results when combining the predictors based on exceptionality measures.
} 
to investigate if this observation holds for other exceptionality measures.

We continued the experiments on the other exceptionality measures hoping to get more insight into the trend observed for typicality. Indeed, Figure 6 (same as Figure 4 but using the CPS instead of typicality) shows the same trend: IB1-IG is worse than Ripper when predicting exceptional instances and it is better when predicting typical instances. The accuracy curves of the two predictors seem to cross at a CPS value of 0.5 , which corresponds to boundary instances. Undefined CPS values (0/0) are assigned a value above 1 (the rightmost point on the graph). Ripper was the one that offered higher accuracy in predicting instances with undefined CPS value for almost all datasets (although not in Figure 6). The result holds for all our datasetfeature set combinations.

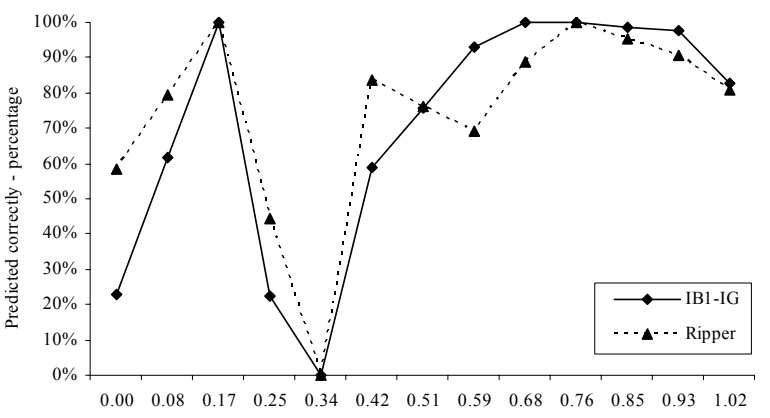

CPS

Figure 6. Percent of instances predicted correctly by IB1-IG and Ripper based on instance CPS (ISCORR dataset with all features) ${ }^{5}$

The experiments with local typicality yield the same results: Ripper constantly outperforms IB1-IG for exceptional instances and they switch places for typical instances (see Figure 7). Again, the accuracy curves cross at boundary instances (local typicality value of 1) and the same observation holds for all dataset-feature set combinations.

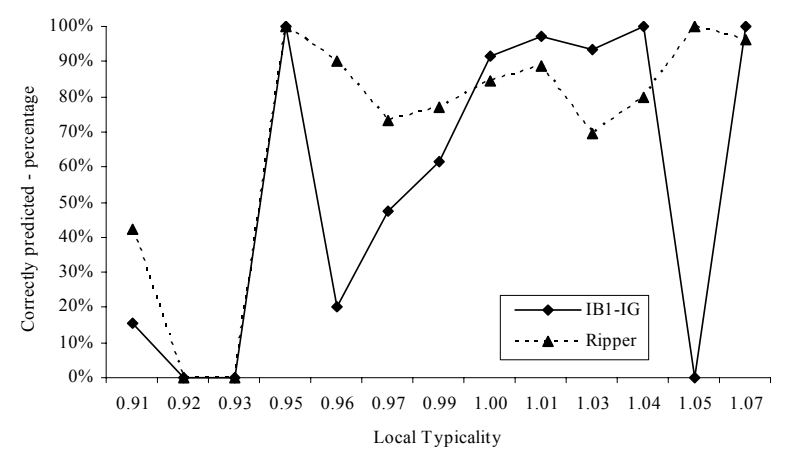

Figure 7. Percent of instances predicted correctly by IB1-IG and Ripper based on instance local typicality (ISCORR dataset with all features)

\footnotetext{
${ }^{5}$ Abrupt movements in curves are caused by small number of instances in that class. We expect that a larger dataset will smooth our graphs.
}

We computed what could be the reduction in error rate if we were to employ both predictors and decide between them based on the instance exceptionality measure. In other words, Ripper prediction was used for exceptional instances and for the left-hand side boundary instances (CPS less than 0.5; typicality less than 1; local typicality less than 1); otherwise IB1-IG prediction was used. The lower bound of this reduction is when we perfectly know which of the predictors offer the correct prediction (in other words the error rate is the number of times both learners furnished wrong predictions). Figure 8 plots the reduction in error rate achieved when deciding between predictors based on typicality, CPS, local typicality and perfect discrimination. The reduction is relative to the best performer on that task. While discriminating based on typicality offered no improvement relative to the best performer, CPS was able to constantly achieve improvement and local typicality improved in six out of eight cases. CPS improved the error rate of the best performer by decreasing it by $1.33 \%$ to $3.18 \%$ (absolute percentage). In contrast with CPS, local typicality offered, for the cases when it improved the accuracy, more improvement decreasing the error rate by up to $4.94 \%$ (absolute percentage). A possible explanation of this difference can be the fact that local typicality captures much more information than CPS (vicinity-level information compared with information very close to the instance).

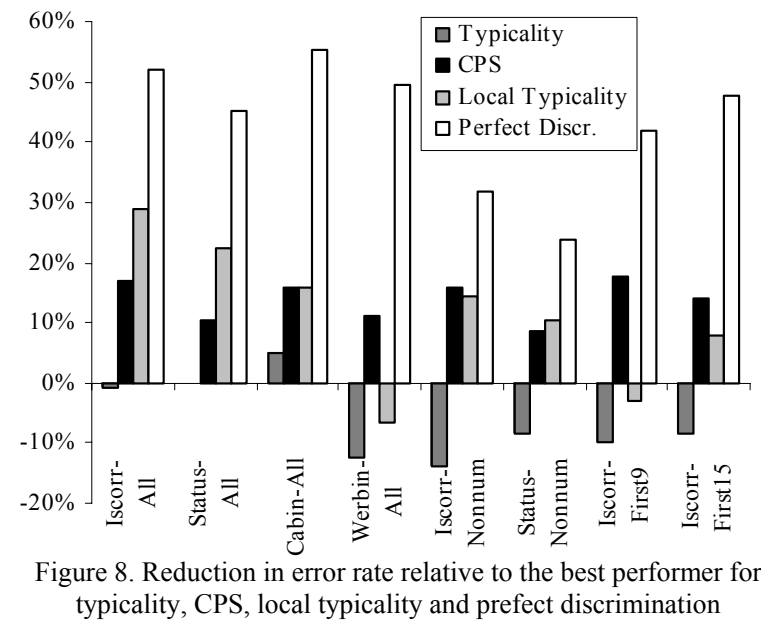

In summary, all our exceptionality measures show the same trend in predicting ability: Ripper performs better than IB1-IG on exceptional instances while IB1IG performs better than Ripper on typical instances. While the fact that IB1-IG does better on typical instances may be linked to its ability to handle subregularities, we have no interpretation for the fact that Ripper does better on exceptional instances. We plan to address this by future work that will look at the distance between exceptional instances and the instances that generated the rule that made the correct prediction for those exceptional instances. 


\subsection{Current directions}

The previous section showed that we can improve the overall accuracy on our datasets if we combine the prediction generated by our learners based on the exceptionality measure of the new instance. Unfortunately, all our exceptionality measures require the class of the instance. Moreover, for binary classification tasks, since all exceptionality criteria are a ratio, changing the instance class will turn an exceptional instance into a typical instance.

To move our results from offline to online, we considered interpolating the exceptionality value for an instance based on its neighbors' exceptionality values (the neighbors from the training set). We performed a very simple interpolation by using the exceptionality value of the closest neighbor (relative to equation (1)). While previous observations are not obvious anymore in online graphs (there is no clear crossing at boundary instances), there is a small improvement over the best predictor. Figure 9 shows that even for this simple interpolation there is a small reduction in almost all cases in error rate relative to the best performer when using online CPS (interpolated CPS).

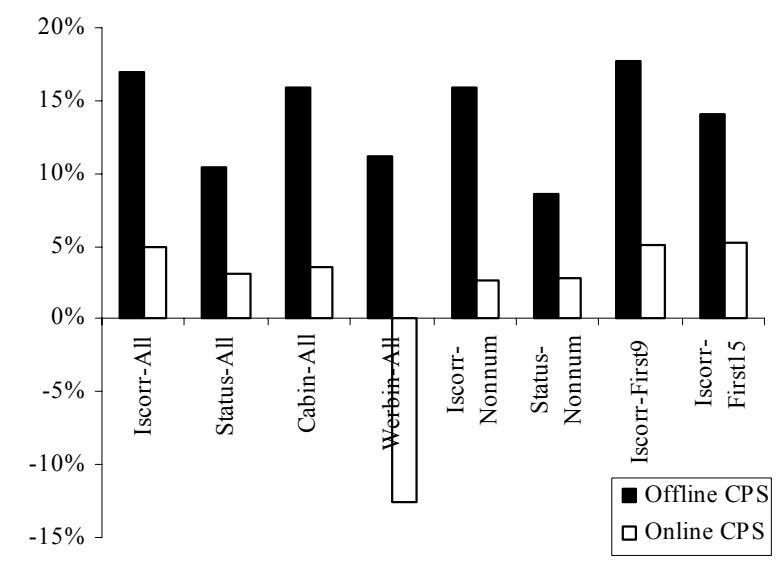

Figure 9. Reduction in error rate relative to the best performer for offline CPS and online CPS

We are currently investigating more complicated interpolation strategies like learning of a model from the training set that will predict the exceptionality value of an instance based on its closest neighbors.

\section{Conclusions}

In this paper we attempted to generalize the results of a previous study to a new set of language learning tasks from the area of spoken dialog systems. Our experiments indicate that previous results do not generalize so obviously to the new tasks. Next, we showed that some exceptionality measures can be used as means to improve the prediction accuracy on our tasks by combining the prediction of our learners based on measures of instance exceptionality. We observed that our memory- based learner performs better than the rule-based learner on typical instances and they exchange places for exceptional instances. We also showed that there is potential for moving these results from offline to online by performing a simple interpolation. Future work needs to address more complicated methods of interpolation, comparison between our method and other attempts to combine rule-based learning and memory-based learning (Domingos, 1996; Golding and Rosenbloom, 1991), comparison with ensemble methods, and whether the results from this paper generalize to other spoken dialog corpora.

\section{Acknowledgements}

We would like to thank Walter Daelemans and Antal van den Bosch for starting us on this work.

\section{References}

William Cohen. 1995. Fast effective rule induction. ICML.

Walter Daelemans, Antal van den Bosch, and Jakub Zavrel. 1999. Forgetting exceptions is harmful in language learning. Machine Learning 1999, 34 :11-43.

Walter Daelemans, Jakub Zavrel, Ko van der Sloot, and Antal van den Bosch. 2001. TiMBL: Tilburg Memory Based Learner, version 4.1, Reference Guide. ILK Technical Report - ILK 01-04.

Pedro Domingos. 1996. Unifying Instance-Based and RuleBased Induction. Machine Learning 1996, 24:141-168

Andrew R. Golding and Paul S. Rosenbloom. 1991. Improving Rule-Based Systems Through Case-Based Reasoning. Proc. AAAI.

Julia Hirschberg, Diane J. Litman, and Marc Swerts. 2001. Identifying User Corrections Automatically in Spoken Dialogue Systems. Proc. NAACL.

Diane J. Litman, Julia Hirschberg, and Marc Swerts. 2000. Predicting Automatic Speech Recognition Performance Using Prosodic Cues. Proc. NAACL.

Diane J. Litman, Julia Hirschberg, and Marc Swerts. 2001. Predicting User Reactions to System Error. Proc. ACL.

Diane J. Litman, Shimei Pan. 2002. Designing and Evaluating an Adaptive Spoken Dialogue System. User Modeling and User-Adapted Interaction, 12(2/3):111-137.

Salzberg, S. 1990. Learning with nested generalised exemplars. Kluwer Academic Publishers.

Elizabeth Shriberg, Rebecca Bates, Paul Taylor, Andreas Stolcke, Klaus Ries, Daniel Jurafsky, Noah Coccaro, Rachel Martin, Marie Meteer, and Carol Van Ess-Dykema. 1998. Can prosody aid the automatic classification of dialog acts in conversational speech?. Language and Speech 41:439—487.

Jianping Zhang. 1992. Selecting typical instances in instance-based learning. Proc. ICML, 470-479. 